Centre in collaboration with the British Association for Accident and Emergency Medicine, June 1995.

3 Underhill TJ, Greene MK, Dove AF. A comparison of the efficacy of gastric lavage, ipecacuanha and activated charcoal in the emergency management of paracetamol overdose. Arch Emerg Med 1990;7:148-54.

\section{The authors reply}

We are grateful for the opportunity to respond to these comments regarding our paper.

With regard to the suggestion that we implied gastric lavage to be an inappropriate measure in scenarios 1 and 2, we must stress that the intention of our paper was to provoke debate (successfully it would appear) rather than to suggest management guidelines.

The paper by Underhill et al (reference 3 above) showed paracetamol levels falling by a mean of $39.3 \%$ over the two hours following lavage. This does not demonstrate causality, particularly in the absence of an adequate control group. The paper also demonstrated a mean fall in serum paracetamol levels of $40 \cdot 7 \%$ following administration of ipecacuana and of $52.5 \%$ over the same two hour period following administration of activated charcoal. The difference between results for lavage and ipecacuana was not significant. The fall in paracetamol levels following charcoal was significantly greater than both other forms of treatment. The paper concludes that "activated charcoal was more effective at limiting absorption of paracetamol following overdose than either gastric lavage or ipecacuana induced emesis". The authors also comment that gastric lavage is not a risk-free procedure.

The new guidelines for the management of paracetamol poisoning recommend lavage or charcoal rather than lavage with charcoal as the optimal treatment within two hours of ingestion (reference 2 above).

I GREAVES GOODACRE P GROUT

Accident and Emergency Department, St Fames's University Hospital,

\section{Support surfaces}

EDITOR,-I was concerned to read the paper by $P$ W Main and M E Lovell entitled "A review of seven support surfaces with emphasis on their protection of the spinally injured". 1

I would not in any way doubt their findings on the pressure problems related to the use of long spinal boards. Unfortunately, however, they seem to have missed the whole point of the use of spine boards in the prehospital care of critically injured patients. Although the spine board may provide a surface for in-line immobilisation of the spine, its primary function is in the road traffic accident setting, where it is used to extricate patients from vehicles and for their subsequent transportation to hospital.

The spine board is the only tool that can be used to slide a patient with a serious injury from a vehicle with safe in-line minimal immobilisation of the spine and retain that immobilisation on route to hospital. With the use of a board for both rearward and side extrication from a vehicle, the patient can be extricated with support to the whole spine safely from virtually any vehicle accident. The board's construction, specifically designed with a slippery surface to slide patients from the wreck, has a major advantage in prehospital care as an extrication device. This is not possible with a vacuum mattress or scoop types of stretcher or, in fact, any other type of stretcher available.

The patient on extrication is immediately immobilised with head and neck restraint and four body straps and transferred to an ambulance trolley. The patient is then transported on the board during the shor transfer to hospital, where, again, the advantage of being on a board is obvious. In the case of a multiple injury patient, rapid transfer from an ambulance to hospital trolley is essential and this is facilitated by rapid transfer on the spine board, again with a patient fully immobilised. The hospital staff, once appraised of the mechanism of injury and apparent injuries to the patient, can decide whether to maintain the patient on the board or transfer them with an appropriate spinal lift to a vacuum mattress.

If a vacuum mattress were available on all front line ambulances, a single ambulance crew would not be able to transfer a patient, once extricated from a wreckage, from a spine board to a vacuum mattress. The vacuum mattress, therefore, although an ideal $A \& E$ department and secondary transfer tool, has number of practical limitations in its prehospital use as a primary stretcher. The spine board certainly does have its limitations, with pressure area problems if patients are left on the board for long periods of time, but its value as an extrication device, enabling extrication with in-line spinal immobilisation for transfer to hospital, cannot be overemphasised.

This paper clearly emphasises the potential hazards of a spine board to a patient, with defined spinal column injury, but one must remember that the majority of patients are placed on a board with only suspected injury, because of their injury pattern or injury mechanism that puts the spine at risk. The safe extrication of a patient from the wreckage is almost certainly of more importance to the integrity of a damaged spinal column than a smaller risk of pressure area problems in the short transfer time to hospital.

\section{J CARNEY} Staffordshire Ambulance Service NHS Trust

1 Main PW, Lovell ME. A review of seven support surfaces with emphasis on their protection of the spinally injured. $f$ Accid Emerg Med 1996;13:34-7.

\section{The authors reply}

Thank you for the opportunity to answer Dr Carney's points. We would value any debate of this most important subject.

We disagree that the long spinal board's main and most used function is in the extrication setting, involving motor vehicle trauma. The spinal board will be used for any patient when spinal injury is suspected, including motorcycle accidents, falls from heights, pedestrian RTAs, diving accidents, etc. We wonder what percentage of calls that an ambulance crew attends and where a spinal board is used are for extrication. We suspect that it is few. It would be helpful if such information were collected, since no direct figures are available. We are grateful to Mr DA Boot (Mersey Trauma Outcome
Study) for providing information from the study. This database, compiled from clinical notes, reveals that of the 658 patients with a trauma score greater than 15 , only 51 patients were recorded as trapped and requiring extrication (personal communication).

If thought necessary, a single ambulance crew could transfer a patient from a spinal board simply by use of a scoop stretcher placed between the patient and the board and then lowered onto a vacuum mattress. This, however, would be time consuming and probably not warranted; it would add time to the evacuation of the casualty.

As covered in our paper, the spinal board is not an ideal surface The spine is not flat! The neck is extended on the board ${ }^{1}$; it causes patients without spinal injury pain and discomfort; it causes pressure sores in those patients with (often irreversible) spinal injury, who may stay on the board until they arrive at a spinal centre. Patients are left on the board longer than necessary due to caution about causing or extending an injury. This is usually until a radiological series is performed. These $x$ rays may also be needed because of pain caused by lying on a board, which cannot be differentiated from significant trauma. ${ }^{2}$ We do not think that in most settings rapid removal from the board takes place, and many casualty departments own boards to continue this type of spinal immobilisation.

Although we do not expect change in practice from our paper we wish to highlight the above points and agree with Dr Carney's suggestion that spinal boards should only be used for the short periods of transfer to hospital from the scene of the accident.

P W MAIN 208 (Merseyside) Field Hospital, $R A M C(V)$ Chavasse House. Sarum Road Liverpool; $L 252 X P$

1 Schringer DL, Larmon B, LeGassick $T$, Blinman T. Spinal immobilisation on a flat board: does it result in a neutral position of the cervical spine. Ann Emerg Med 1991; 20:878-81.

2 Chan D, Goldberg R, Tascone A, Harmon S, Chan L. The effect of spinal immobilisation on healthy volunteers. Ann Emerg Med 1994;23:48-51.

\section{Fast tracking patients with a proximal} femoral fracture

EDITOR,-Ryan et al are right to highlight the need for improvement in the management of patients with proximal femoral fractures in accident and emergency departments in the United Kingdom ${ }^{1}$ but even with the fast tracking system in place, over $75 \%$ of their patients waited two hours or more in the A\&E department before transfer. Consequently, we feel that the system does not fully address the real priorities for treatment of such patients. We define these priorities as (a) the urgent provision of effective analgesia and splintage, (b) prompt imaging and other investigations to allow for a plan of action (c) the primary prevention of common complications such as pressure sores, and (d) clear and frequent communication with the patient (and carers) about the likely timescale of transfer to a ward, surgery and postoper- 